\title{
Quantitative trait loci for carcass, internal organ and meat quality traits on porcine chromosomes 16, 17 and 18
}

\author{
Débora M. Paixão, Miguel I. da Silva Filho, Mário S. Pereira, Marcos S. Lopes, Leandro Barbosa, \\ Katiene Régia Silva Souza, Paulo S. Lopes and Simone E.F. Guimarães \\ Departamento de Zootecnia da Universidade Federal de Viçosa, Viçosa, MG, Brazil.
}

\begin{abstract}
The objective of this study was to map quantitative trait loci (QTL) on porcine chromosomes 16,17 and 18 and to determine their association with carcass, organ and meat quality traits. An F2 population was produced by crossing two boars of the naturalized Brazilian Piau breed with 18 commercial females (Landrace $\mathrm{x}$ Large White $\mathrm{x}$ Pietrain). The population was genotyped for 11 microsatellite markers distributed over the three chromosomes and the results were used to construct a marker-specific linkage map for the population. Analysis of the polymorphic information content showed that the microsatellite markers were adequate for the study of quantitative traits. QTL were identified by regression interval mapping using QTL Express software. QTL not previously described in the literature were detected on chromosome 16, whereas QTL described in other populations were detected on chromosomes 17 and 18 . The information from the significant QTL identified here will be useful for future fine-mapping studies and should provide a better understanding of productive phenotypes in pigs.
\end{abstract}

Key words: divergent crosses, microsatellite markers, pig production, QTL mapping.

Received: November 12, 2007; Accepted: April 18, 2008.

Quantitative traits (almost all traits of economic interest) are controlled by several genes and are influenced by the environment. One approach for determining the approximate number of loci involved in the genetic control of quantitative traits and how these loci are distributed in the genome, as well as the intensity of their effects, is to study them indirectly by associating them with genetic markers (Dekkers and Hospital, 2002).

The identification of quantitative trait loci (QTL) in domestic animals is facilitated by the accumulation of mutations produced over many years of selection at loci that influence phenotypic traits (Andersson and Georges, 2004). Mackay (2001) stated that if there is a QTL-marker linkage then differences in the mean values of a quantitative trait will be observed among individuals with different genotypes for this marker. Moreover, the identification of genes may increase the efficiency of selection in animal breeding, especially in cases of low heritability traits or traits that can only be measured after slaughter, such as meat quality and some carcass traits.

The main purpose of the present study was to map the QTL for carcass, internal organ and meat quality traits located on porcine chromosomes 16 (SSC16), 17 (SSC17)

Send correspondence to Simone Eliza Facioni Guimarães. Departamento de Zootecnia, Universidade Federal de Viçosa, 36570-00 Viçosa, MG, Brazil. E-mail: sfacioni@ ufv.br. and 18 (SSC18) in an F2 population produced by crossing naturalized Piau boars and commercial females (Landrace $\mathrm{x}$ Large White $\mathrm{x}$ Pietrain).

Details of the experimental F2, the phenotypes and the DNA extraction procedures used have been described by Band et al. (2005a,b) and Faria et al. (2006). Eleven primer pairs for microsatellite markers distributed on SSC16 (S0006, SW977, SW2517, SW1897), SSC17 (SW24, SW2142, S0359, SW2427) and SSC18 (SW1984, S0120, S0177) were used. Amplifications were done in an MJ Research PTC 100-96 $6^{\circledR}$ thermocycler, according to standard laboratory procedures. The amplified fragments were scored automatically by GenScan software installed in an ABI PRISM 310 sequencer (Applied Biosystems). Annotation and genotype checking were done manually by two independent and previously trained technicians.

The allele frequency ( $\rho$ i), expected $(\mathrm{He})$ and observed heterozygosity (Ho), and polymorphic information content (PIC) for the microsatellite markers were calculated for 20 parental animals, 64 F1 animals and approximately 500 F2 animals by using the CERVUS software, version 2.0 (Marshall et al., 1998). CRIMAP software was used to construct linkage maps of SSC16, SSC17 and SSC18. The resulting genotypic and phenotypic files and linkage maps were analyzed with QTL Express software (Seaton et al., 2002). 
The statistical model used assumed that QTL were bi-allelic, with fixed alternative alleles in each parental breed. The probability that each F2 individual presents each of the three QTL genotypes was calculated according to the genotype of the markers at 1-cM intervals along the chromosome. These probabilities were used for regression of the traits on additive and dominance coefficients of the QTL studied for each animal, as described by Haley et al. (1994). The chromosome-wide significance thresholds $(\alpha=0.05$ and $\alpha=0.01)$ were determined using QTL Express software, with 10,000 permutations per test (Churchill and Doerge, 1994). The genome-wide significance threshold $(\alpha=0.05)$ was obtained using Bonferroni's correction (Knott et al., 1998). The 95\% confidence interval $(95 \% \mathrm{CI})$ for localization of the QTL was calculated using the chi-square approximation $\left(X^{2}\right)$ as described by PérezEnciso et al. (2000). The following statistical model was adopted:

$$
y_{i j k l}=S_{i}+L_{j}+H_{k}+\left(C_{i j k l}-\bar{C}\right) b+c_{a} a+c_{d} d+e_{i j k l}
$$

where $y_{i j k}=$ phenotype; $S_{i}=$ fixed effect of sex $i, i=1$ (male), 2 (female); $L_{j}=$ fixed effect of batch $j, j=1,2,3,4$, 5; $H_{k}$ = fixed effect of the halothane genotype $k, k=1(\mathrm{NN})$, $2(\mathrm{Nn}) ;\left(C_{i j k}-\bar{C}\right) b=$ adjustment for covariables; $c_{a}=P(Q Q / M i)-P(q q / M i) ; c_{d}=P(Q q / M i)$.

The halothane genotype was included as a fixed effect since Band et al. (2005a,b) reported significant effects of the $\mathrm{Hal}^{1843}$ mutation on performance, carcass and meat quality traits in this population. Carcass weight at slaughter was used as a covariable for carcass and organ traits, and age at slaughter was used as a covariable for meat quality traits.
The additive fraction of phenotypic variance $\left(h_{Q}^{2}\right)$ in the F2 generation explained by a given QTL was computed according to Pérez-Enciso et al. (2000), assuming that alternative alleles are fixed in each breed: $h_{Q}^{2}=a^{2} / 2 \sigma_{\mathrm{Y}}^{2}$, where $\sigma_{\mathrm{Y}}$ is the residual standard deviation for fixed effects and covariables, except for the QTL, and $a$ is the additive effect generated by QTL Express software. $c_{a}$ and $c_{a}$ are conditional probability functions of the QTL given the genotype of the markers, where $P(Q Q)$ is the probability that the QTL alleles are homozygous and originated from the naturalized Piau breed, $\mathrm{P}(\mathrm{qq})$ is the probability that the QTL alleles are homozygous and originated from the commercial breed, and $\mathrm{P}(\mathrm{Qq})$ is the probability that the QTL alleles are heterozygous.

Evaluation of the amplified loci on SSC16 provided PIC (Polymorphic information content) values of 0.67 , 0.54, 0.50 and 0.32 for loci S0006, SW977, SW2517 and SW1897, respectively. On SSC17, the PIC values were $0.52,0.54,0.59$ and 0.61 for loci SW24, SW2142, S0359 and SW2427, respectively. On SSC18, PIC values of 0.69, 0.48 and 0.60 were obtained for loci SW1984, S0120 and S0177, respectively. The total length of the maps constructed was $166 \mathrm{cM}$ for SSC16, $151 \mathrm{cM}$ for SSC17 and $74 \mathrm{cM}$ for SSC18. Estimates of additive, dominance and imprinting effects were obtained for all traits, although in no case were the estimates of the imprinting effect significant. The significant QTL obtained for the three chromosomes analyzed are summarized in Table 1.

Analysis of the PIC values showed that all of the markers used were efficient for the analysis of quantitative traits since the applicability of these markers was directly

Table 1 - Maximum F statistics $\left(\mathrm{F}_{\max }\right)$, number of animals analyzed for each trait $(\mathrm{N})$, chromosomal position of each QTL, confidence interval $(\mathrm{CI})$, and estimates of additive and dominance effects and standard errors (SE) for significant traits on SSC16, 17 and 18.

\begin{tabular}{|c|c|c|c|c|c|c|c|}
\hline Trait & $\mathrm{N}$ & Position in $\mathrm{cM}(\mathrm{CI})$ & $\mathrm{F}_{\operatorname{Max}}$ & \multicolumn{2}{|c|}{ Additive (SE) } & \multicolumn{2}{|c|}{ Dominance (SE) } \\
\hline \multicolumn{8}{|c|}{ SSC16 } \\
\hline Bacon depth (mm) & 528 & $0(0-23)$ & $5.33 * *$ & -0.15 & $(0.05)$ & -2.03 & $(0.72)$ \\
\hline $\begin{array}{l}\text { Midline lower Backfat thickness } \\
\text { above the last lumbar vertebrae (mm) }\end{array}$ & 531 & $16(0-37)$ & $5.21 * *$ & -2.02 & $(0.81)$ & -4.98 & $(1.46)$ \\
\hline Heart weight (kg) & 532 & $93(68-145)$ & $5.52 * *$ & 0.009 & $(0.003)$ & 0.009 & $(0.008)$ \\
\hline Lung weight (kg) & 526 & $113(88-136)$ & $4.91^{*}$ & 0.01 & $(0.01)$ & 0.003 & $(0.01)$ \\
\hline Redness (HUNTER) & 446 & $28(6-44)$ & $8.8^{* * *}$ & 0.18 & $(0.10)$ & 0.50 & $(0.22)$ \\
\hline Cooking loss $(\%)$ & 501 & $51(29-91)$ & $4.05^{*}$ & -0.53 & $(0.27)$ & -1.49 & $(0.51)$ \\
\hline \multicolumn{8}{|c|}{$\mathrm{SSC} 17$} \\
\hline $\begin{array}{l}\text { Backfat thickness at last rib, } 6.5 \mathrm{~cm} \\
\text { from the midline }(\mathrm{mm})\end{array}$ & 531 & $90(79-109)$ & $4.54^{*}$ & 0.85 & $(0.31)$ & 1.15 & $(0.44)$ \\
\hline \multicolumn{8}{|c|}{$\mathrm{SSC} 18$} \\
\hline $\begin{array}{l}\text { Backfat thickness at last rib, } 6.5 \mathrm{~cm} \\
\text { from the midline }(\mathrm{mm})\end{array}$ & 531 & $65(16-74)$ & $4.07 *$ & 0.89 & $(0.30)$ & -0.76 & $(0.65)$ \\
\hline Total loss (\%) & 501 & $0(0-54)$ & $4.47^{*}$ & 0.50 & $(0.27)$ & 0.09 & $(0.38)$ \\
\hline
\end{tabular}

$*, * *$ and $* * *$ significant at $5 \%$ and $1 \%$ chromosome-wide level, and at $5 \%$ genome-wide level, respectively. 
related to their degree of polymorphism. Except for the markers SW1897 (0.32) located on SSC16 and S0120 $(0.48)$ located on SSC18, which were classified as moderately polymorphic, all of the other markers were considered to be highly polymorphic, with a PIC $>0.5$ (Botstein et al., 1980).

The lengths of the linkage maps differed from those reported in the literature. This was expected since a different set of markers and different populations were used. The genetic diversity within these populations influences the recombination rate (meiosis) and the CRIMAP program uses this information as a basis for the construction of linkage maps, a fact reflected in the variation in linkage map length among populations. The most likely source of differing map lengths is the presence of undetected genotyping errors that result in double recombinations in the CRIMAP analysis. The linkage map lengths (CRIMAP) reported for SSC16, SSC17 and SSC18 in other studies are 110124.2 cM (Bidanel et al., 2001; Pierzchala et al., 2003), 90-97.8 cM (Bidanel et al., 2001; Pierzchala et al., 2003) and 48.7-89 cM (Bidanel et al., 2001; Dragos-Wendrich et al., 2003), respectively.

The QTL associated with bacon depth (BCD) was located between the S0006 and SW977 markers, the same region where the QTL for lower midline lower backfat thickness above the last lumbar vertebrae (L) was found. These QTL were situated in the initial portion of SSC16, indicating the existence of gene(s) that influence both traits in this region. In the case of significant QTL for traits related to fatness deposition, $\mathrm{BCD}$ (additive effect $=-0.05$ ) and $\mathrm{L}$ (additive effect $=-2.02$ ), the negative additive effect of the QTL implies that the alleles of the commercial line cause a decrease in these phenotypes (Table 1). There are no literature reports of QTL for internal organs on SSC16. Hence, the finding that SSC16 contained QTL for the weight of internal organs such as heart and lung (Table 1) was novel.

The QTL detected on SSC16 for HUNTER redness of the meat (A) is interesting because this locus is directly related to meat color, one of the main aspects used by consumers when purchasing fresh meat, and was significant at the genome-wide level (Figure 1). The genome-wide level of significance is used to detect the presence of QTL somewhere within the genome and controls for the rate of type I (false-positive) errors (Churchill and Doerge, 1994). The positive additive effect $(0.18)$ of the QTL for A implies that alleles of the Piau breed increase this phenotype. Animals of this breed are appreciated because of the good organoleptic aspects of their meat, which disagrees with the negative additive effect $(-0.53)$ of the QTL for cooking loss (CL), indicating that the alleles of the commercial line result in a decrease of this phenotype (Table 1). From an industrial point of view, cooking loss is related to the waterholding capacity of meat, an essential trait for storage and processing that influences the yield and color of cured meat products (Van Oeckel et al., 1999).

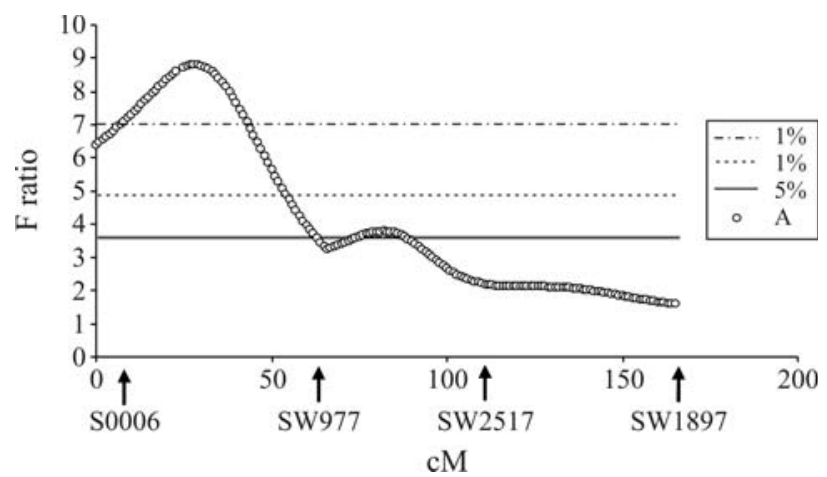

Figure 1 - Estimates of the $\mathrm{F}$ ratio for meat redness (A). The horizontal lines indicate the levels of chromosome-wide $(5 \%=$ continuous line, $1 \%=$ dashed line $)$ and genome-wide $(5 \%=$ dot-dashed line $)$ significance.

The positive additive effect (0.85) of the QTL for backfat thickness at last rib $6.5 \mathrm{~cm}$ from the midline (P2) (located on SSC17) implies that the alleles of the Piau breed increase this phenotype (Table 1), and agrees with the fact that this breed is known as a fat type pig. QTL related to fat deposition were also identified on SSC17 in an experimental population resulting from the crossing of Meishan and Pietrain pigs (Pierzchala et al., 2003). Another QTL for P2 was detected on SSC18 but was poorly delimited in its confidence interval (Table 1). This finding suggests that the definition of this QTL region may be improved by using a larger number of markers.

With respect to meat quality traits on SSC18, a QTL for total loss was identified. However, it was not expected that the genotype of the Piau breed would contribute to an increase of the total loss phenotype (additive effect $=0.27$ ) (Table 1) since the meat of this breed generally has better organoleptic properties than that of commercial breeds. A QTL for drip loss on SSC18 (24 cM) has previously been reported by De Koning et al. (2001) in a population resulting from the crossing of Meishan animals and pigs of commercial breeds.

Molecular information becomes even more useful when associated with low heritability traits and/or traits that require slaughtering of the animal for their evaluation. In the present study, QTL related to some of these traits (BCD, L, P2, A, CL and TL) were identified. In addition, QTL (CL, A, BCD, HEART and LUNG) not previously found on SSC16 were detected on this chromosome. QTL described in other populations were also identified here, including backfat thickness at last rib $6.5 \mathrm{~cm}$ from the midline on SSC17, and backfat thickness at last rib $6.5 \mathrm{~cm}$ from the midline and total loss on SSC18. Analysis of these newly identified QTL should allow the identification of phenotype-related genes and provide a better understanding of these traits. Finally, the inclusion of additional markers in QTL regions with a poorly delimited confidence interval will provide a better definition of the $\mathrm{F}$ curve and greater accuracy in the identification of genes. 


\section{Acknowledgments}

We thank CAPES, FAPEMIG, CNPq and FINEP for financial support, and Prof. Max F. Rothschild, coordinator of the US Pig Genome Project, for donating the fluorescent primers.

\section{References}

Andersson L and Georges M (2004) Domestic-animal genomics: Deciphering the genetics of complex traits. Nat Rev Genet 5:202-212.

Band GO, Guimarães SEF, Lopes PS, Schierholt AS, Silva KM, Pires AV, Benevenuto Júnior AA and Gomide LAM (2005a) Relationship between the Porcine Stress Syndrome gene and pork quality trait in F2 pigs resulting from divergent crosses. Genet Mol Biol 28:88-91.

Band GO, Guimarães SEF, Lopes PS, Peixoto JO, Faria AF, Pires AV, Figueiredo FC, Nascimento CS and Gomide LAM (2005b) Relationship between the Porcine Stress Syndrome gene and carcass and performance trait in F2 pigs resulting from divergent crosses. Genet Mol Biol 28:92-96.

Bidanel JP, Milan D, Iannuccelli N, Amigues Y, Boscher MY, Bourgeois F, Caritez JC, Gruand J, Le Ro P, Lagant H, et al. (2001) Detection of quantitative trait loci for growth and fatness in pigs. Genet Sel Evol 33:289-309.

Botstein D, White RL, Skolnick M and Davis RW (1980) Construction of genetic linkage map in man using restriction fragment length polymorphisms. J Hum Genet 32:314-331.

Churchill GA and Doerge RW (1994) Empirical threshold values for quantitative trait mapping. Genetics 138:963-971.

Dekkers JCM and Hospital F (2002) The use of molecular genetics in improvement of agricultural populations. Nat Rev Genet 3:22-32.

De Koning DJ, Rattink AP, Harlizius B, Van Arendonk JA, Brascamp EW and Groenen MAN (2001) Detection and characterization of quantitative trait loci for meat quality traits in pigs. J Anim Sci 79:2812-2819.

Dragos-Wendrich M, Stratil A, Hojny J, Moser G, Bartenschlager H, Reiner G and Geldermann H (2003) Linkage and QTL mapping for Sus scrofa chromosome 18. J Anim Breed Genet 120:138-143.
Faria DA, Guimarães SEF, Lopes PS, Pires AV, Paiva SR, Sollero BP and Wenceslau AA (2006) Association between G316A growth hormone polymorphism and economic traits in pigs. Genet Mol Biol 29:634-640.

Haley CS, Hill WG, Baret PV, Hetzel DJS and Nicholas FW (1994) Detection and mapping of quantitative trait loci in farm animals. Livest Prod Sci 52:135-144.

Knott SA, Marklund L, Haley CS, Andersson K, Davies W, Ellegren H, Fredholm M, Hansson I, Hoyheim B, Lundstrom K, et al. (1998) Multiple marker mapping of quantitative trait loci in a cross between outbred wild boar and Large White pigs. Genetics 148:1069-1080.

Mackay TFC (2001) Quantitative trait in Drosophila. Nat Rev Genet 2:11-20.

Marshall TC, Slate J, Kruuk LEB and Pemberton JM (1998) Statistical confidence for likelihood-based paternity inference in natural populations. Mol Ecol 7:639-655.

Pérez-Enciso M, Clop A, Noguera JL, Óvilo C, Coll A, Folch JM, Babot D, Estany J, Oliver MA, Díaz I, et al. (2000) A QTL on pig chromosome 4 affects fatty acid metabolism: Evidence from an Iberian by Landrace intercross. J Anim Sci 78:2525-2531.

Pierzchala M, Cieslak D, Reiner G, Bartenschlager H, Moser G and Geldermann H (2003) Linkage and QTL mapping for Sus scrofa chromosome 17. J Anim Breed Genet 120:132137.

Seaton G, Haley CS, Knott SA, Kearsey M and Visscher PM (2002) QTL Express: Mapping quantitative trait loci in simple and complex pedigrees. Bioinformatics 18:339-340.

Van Oeckel MJV, Warnents N and Bouequé CV (1999) Comparison of different methods for measuring water holding capacity and juiciness of pork versus on-lines screening methods. Meat Sci 51:313-320.

\section{Internet Resources}

Green P, Falls K and Crooks S, CRIMAP Documentation, version 2.4, March 26, 1990. http://linkage.rockefeller.edu/soft/crimap. Associate Editor: Pedro Franklin Barbosa

License information: This is an open-access article distributed under the terms of the Creative Commons Attribution License, which permits unrestricted use, distribution, and reproduction in any medium, provided the original work is properly cited. 